\title{
Seeing the locust in the swarm: accounting for spatiotemporal hierarchy improves ecological models of insect populations
}

\author{
Douglas Lawton, Peter Scarth, Edward Deveson, Cyril Piou, Allan Spessa, Cathy Waters and \\ Arianne J. Cease
}

D. Lawton (https://orcid.org/0000-0003-2363-4084) $\square$ (ddlawton@asu.edu), C. Waters and A. J. Cease (https://orcid.org/0000-0002-4778-0775), School of Life Sciences, Arizona State Univ., Tempe, AZ, USA. DL also at: Dept of Entomology and Plant Pathology, North Carolina State Univ., Raleigh, NC, USA. - P. Scarth, Joint Remote Sensing Research Program, School of Environment and Earth Sciences, Univ. of Queensland, Brisbane, QLD, Australia. - E. Deveson and A. Spessa, Australian Plague Locust Commission, Fyshwick, ACT, Australia. ED also at: Fenner School of Environment and Society, Australian National Univ., ACT, Australia. - C. Piou, CIRAD, UMR CBGP, Univ. Montpellier, CIRAD, INRAE, Inst. Agro, IRD, Montpellier, France. CW also at: NSW Dept of Primary Industries, Dubbo, NSW, Australia. AJC also at: School of Life Sciences Arizona State Univ., Tempe, AZ, USA.

\section{Ecography}

2022: e05763

doi: $10.1111 /$ ecog.05763

Subject Editor: Cory Merow Editor-in-Chief: Miguel Araújo Accepted 10 December 2021
Ecological phenomena operate at different spatial scales and are not uniform across landscapes or through time. One ecological theory that attempts to account for scaling and spatiotemporal variances is hierarchical patch dynamics. It introduces a hierarchical patch network with smaller spatiotemporal scales being nested within larger scales. However, few studies have modeled its presence within animal population dynamics. Locusts are an excellent model for investigating the spatiotemporal hierarchy of animal population dynamics, due to their high migratory capacity, large geographic ranges that extend across widely differing environments, and available long-term data on distributions. Here, we investigated the influence of preceding vegetation growth on desert locust Schistocerca gregaria and Australian plague locust Chortoicetes terminifera outbreaks on three spatial levels (species range $>$ geographic region $>$ land unit) and between seasons. Both species are dryland herbivores with population dynamics linked to habitat productivity pulses after rain. We used NDVI data (MODIS imagery) as a measure of vegetation growth in hierarchical generalized additive models at different scales. Locust outbreaks were either preceded by vegetation growth between 78 and 32 days (Australian plague locusts) or 32 and 20 days before (desert locust) the observation. Although prior vegetation growth characterized outbreaks of both species, the temporal pattern of NDVI differed between spatiotemporal levels. All model selection criteria selected for a similar spatial hierarchy for both species: geographic region $>$ land unit which supports the hierarchical patch dynamics paradigm. Further, it illuminates important timing differences between geographic regions and land units for preceding vegetation growth and locust outbreaks which can help locust managers identify when and where outbreaks occur. By acknowledging the spatiotemporal patterning of locust abundance, we account for heterogeneity of population dynamics throughout species ranges. Our findings demonstrate the importance of incorporating spatiotemporal variation in population models of insects and other animals.

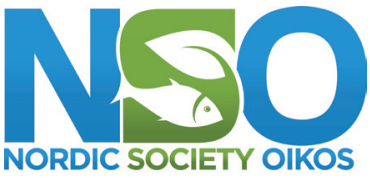

www.ecography.org
(C) 2022 The Authors. Ecography published by John Wiley \& Sons Ltd on behalf of Nordic Society Oikos

This is an open access article under the terms of the Creative Commons Attribution License, which permits use, distribution and reproduction in any medium, provided the original work is properly cited. 
Keywords: grasshoppers, hierarchical generalized additive models, hierarchical patch dynamics, insect outbreaks, remote sensing

\section{Introduction}

The spatiotemporal dynamics of populations has been a central theme in ecology for over a century (Grinnell 1917, Davidson and Andrewartha 1948, Andrewartha and Birch 1954, Macarthur 1958, Hanski 1998). However, there remains wide scope for investigating and understanding the abiotic (e.g. climate, geological variances) and biotic (e.g. food availability) factors affecting the dynamics of each species (Council 2001, Sutherland et al. 2013, Padilla et al. 2014, Stacey 2017). Numerous researchers have shown that ecological phenomena operate on multiple spatiotemporal scales (Senft et al. 1987, Wiens 1989, Levin 1992, Wu and Loucks 1995, Pierson and Turner 1998). For example, caribou and elk populations are distributed by multi-scale resource selections: from individual and herd home ranges to the entire species range (Johnson 1980, Johnson et al. 2004, Coe et al. 2011, Decesare et al. 2012). Schooling fish and krill organize into three levels: individuals congregate in schools or swarms which are concentrated into patches linked to mesoscale environmental features, and finally these patches are aggregated in areas that reflect habitat constraints at range boundaries (Murphy et al. 1988, Fauchald et al. 2000, Fauchald and Erikstad 2002, Fauchald and Tveraa 2006). Since vegetation and consequently animals are heterogeneously distributed because of spatiotemporal variation in climatic and other abiotic factors (Watt 1947, Greig-Smith 1979, Condit et al. 2000, Ives et al. 2008), a spatiotemporal hierarchy of animal population dynamics must be acknowledged.

The hierarchical patch dynamics (HPD) paradigm allows for linkages between spatial and temporal scales through a nested model of patches within patches (Kotliar and Wiens 1990, Wu and Loucks 1995). This paradigm can capture the inherent complexity of landscape level analyses which have emergent properties that arise across different scales (Newman et al. 2019). HPD can integrate multiple scales whereas most current approaches that attempt to address scale, model the levels independent of each other. In a review of multi-scale habitat selection studies, McGarigal et al. (2016) found only $20 \%$ (173 out of 859 ) were quantitative and met their most basic definition of being multi-scale which was modeling multiple levels independently. This can be problematic because many animal populations do not act on single spatiotemporal levels. Here we model the hierarchical nature of outbreaks of two locust species (Fig. 1): the desert locust (Schistocerca gregaria, Forskål 1775) and the Australian plague locust (Chortoicetes terminifera, Walker 1870).

Locusts are grasshoppers that go through periodical population irruptions (i.e. outbreaks) which influence ecosystem functioning (Barbosa et al. 2012) and also pose significant issues to food security globally (Cullen et al. 2017). Therefore, it is important to understand locust population regulation. The majority of locust species live within variable dryland ecosystems which are characterized by the resourcepulse paradigm in which ecosystem productivity dramatically increases with pulses of resources usually connected with weather patterns (Noy-Meir 1973, 1974, Whitford 2002, Schwinning and Sala 2004, Morton et al. 2011). Specifically, precipitation leading to vegetation growth supports increases in locust populations (Chapman and Joern 1990). Most forecasting and predictive models have therefore been driven by some combination of preceding climate including precipitation, soil moisture and vegetation growth, such as the normalized difference vegetation index (NDVI) derived from earth observation satellites (Cressman 2013, Deveson 2013, Piou et al. 2013, Wang et al. 2019, Mangeon et al. 2020).

Several locust species are suitable models for examining scaling issues in animal population dynamics. This is because locust outbreaks develop in response to regional ecological conditions across very large geographic ranges. For example, using an occupancy modeling approach, Veran et al. (2015) showed that Australian plague locust outbreak probability changes spatially and a hierarchical modeling approach can aid in predicting outbreaks. Similarly, desert locust outbreak probability likely varies spatially since this species can outbreak anywhere from West Africa to India (Uvarov 1977), an area encompassing enormous variations in abiotic and biotic variables. Vegetation, which is important for locust populations to build, changes in structure, nutrient availability and community composition between geographical areas and seasons (Watt 1947, Greig-Smith 1979, White 1983). Therefore, accounting for multiple spatial levels via the HPD in a single modeling framework could support hypotheses about locust plague ecology that are untested at local to global scales.

For this study, the spatiotemporal hierarchy of locust outbreaks are broken into four biologically relevant levels (Fig. 1; larger pictures can be found in Supporting information). The 'species range' level is the entire range where outbreaks of each locust species have been observed. At this level, the important factors are migration and large-scale climatic factors (e.g. El Niño Southern Oscillation, Indian Ocean Dipole, Southern Annular Mode and climate change). The second level involves large geographic areas that experience similar climatic conditions (e.g. seasonal rainfall zones). The third level includes bioregions or ecoregions (hereafter termed land units) with similar abiotic and biotic conditions (e.g. soil characteristics, rainfall, vegetation structure, etc.). Further, there is an independent temporal level of season, as all spatial levels vary based on the time of the year due to changing temperature and rainfall. Each level poses a bottleneck which could constrain locust breeding and population increase. Therefore plagues (large-scale outbreaks) can develop when favorable habitat conditions occur over multiple regions throughout multiple seasons.

In this study, we investigate how changes in preceding vegetation growth predicts nymphal outbreaks of two locust 
Australian plague locust

(Chortoicetes terminifera)

(A)
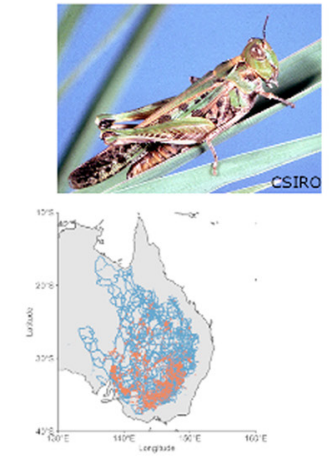

(B)

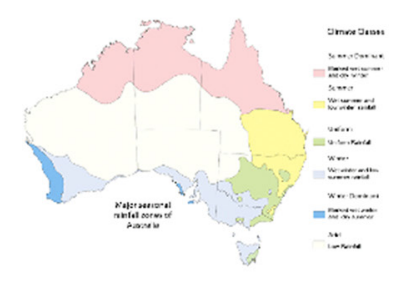

(C)

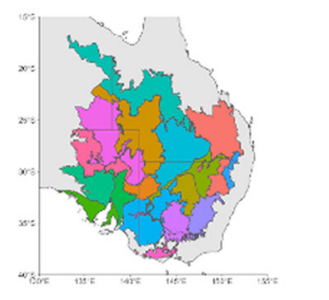

(D)
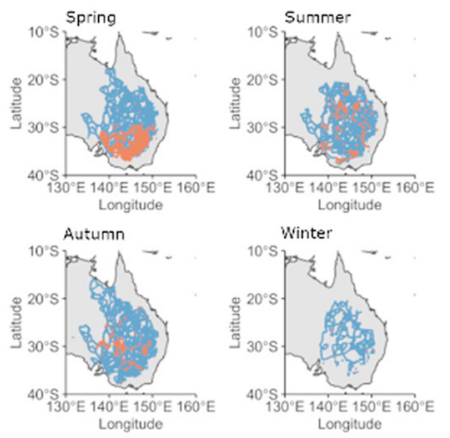

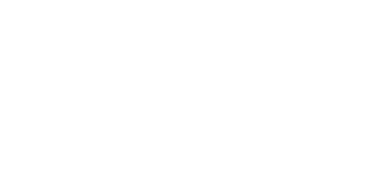

(E)

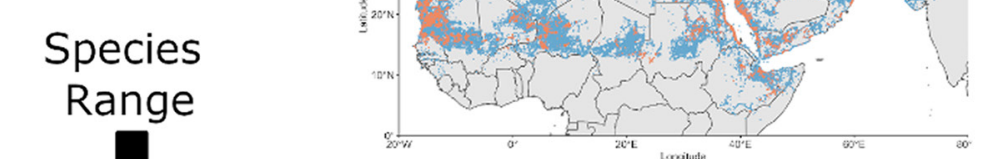

(F)

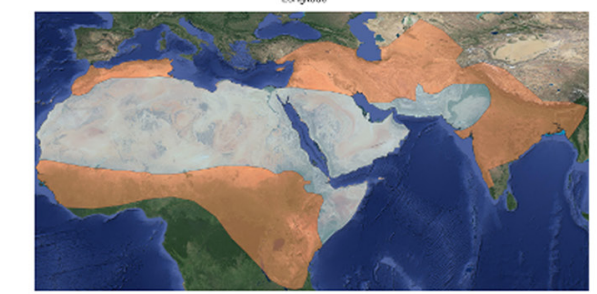

(G)

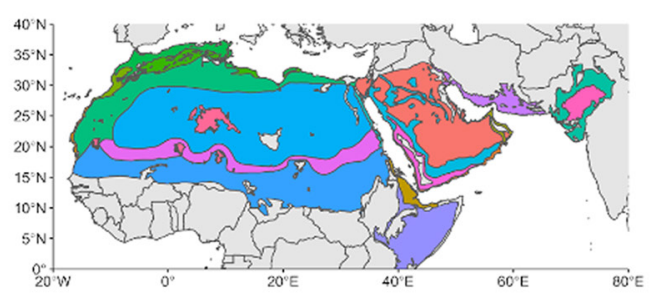

(H)
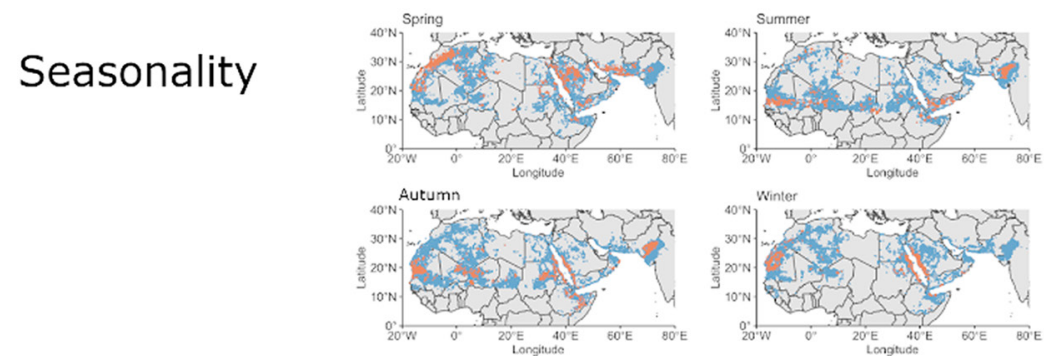

Figure 1. The hierarchical structure of Australian plague locust (A)-(D) and desert locust (E)-(H) ecology. The hierarchy is as follows: species range $(A, E)$, geographic regions which vary based on large scale climatic patterning $(B, F)$, land unit which vary based on abiotic and biotic conditions (C, G). Lastly, seasonality $(\mathrm{D}, \mathrm{H})$ is an important temporal effect on all levels from species range to land unit. For clarity, we have included individual pictures within the Supporting information. Pictures are both under Creative Commons 3.0 license with credit given within.

species that occur on different continents using three, nested spatial (species range $>$ geographic region $>$ land unit) levels and temporally through seasonality (Fig. 1). Since preceding herbaceous vegetation productivity is assumed essential for nymph growth and survival to adulthood, we expect it will be an important factor determining outbreaks. Due to the spatiotemporal variation of biotic and abiotic variables we expect the specific relationships between preceding vegetation growth and outbreaks to be different for each species. However, we expect that the nested spatiotemporal hierarchy will emerge as a crucial characteristic for any outbreak.

\section{Methods}

\section{Locust outbreak data source}

Data for both species were obtained from survey and management operations (e.g. reported outbreaks for control via pesticide spraying) databases. We chose to analyze only nymph data as their presence more closely reflects prior proximal habitat conditions. Since adults are highly mobile and can migrate over long distances, effective locust plague management is focused on predicting and preventing nymphal 
outbreaks to prevent populations from becoming adults (Therville et al. 2021).

As a result of discrepancies in density categorization and identification between observers, and the low detection probability of nymphs at low densities, we decided to identify probable non-outbreaks (e.g. '0') and outbreaks (e.g. '1') similar to Veran et al. (2015). Outbreaks are characterized by high density, gregarious nymphs. This binary threshold differed between species because data collection differed as discussed below.

\section{Australian plague locust}

Australian plague locust data between the years 2000 and 2017 ( -185000 survey or report records) was provided by the Australian Plague Locust Commission and the state agricultural agencies of Queensland, New South Wales, South Australia and Victoria. Records from each agency were adjusted to the same nymph density class schema (Deveson and Hunter 2002). The data structure is georeferenced points with ordinal categorical response values for both adult and nymph densities. The value ranges for nymphs were: $0=$ nil, $1=<5 \mathrm{~m}^{2}, 2=5-30 \mathrm{~m}^{2}, 3=30-80 \mathrm{~m}^{2}, 4=>80 \mathrm{~m}^{2}$. Point distributions can be seen in Fig. $1 \mathrm{~A}$ and a larger picture can be found in Supporting information. We used a binary data schema by selecting density categories $0-2$ as non-outbreaks (e.g. ' 0 ') and categories 3-4, that exhibit gregarious behavior, as successful nymph outbreaks (e.g. ' 1 ').

\section{Desert locust}

Desert locust outbreak data was provided by the Food and Agriculture Organization (FAO) of the United Nations (FAO 2020). FAO stores and cleans (e.g. checks for errors and standardization) the data coming from the affected countries that conduct ground surveys with trained officers and generalized common standards of information reporting. This dataset spanned 19 years from 2000 to 2019, with a total -340000 records. The data structure is georeferenced points that identify whether solitarious, transient or gregarious locusts were present based on morphological characteristics of the individuals observed in each location (Cressman 2001). Distribution of points can be seen in Fig. 1E with a larger map found in Supporting information. If transient or gregarious nymphs were present, the observation was classified as a successful outbreak. Non-outbreak observations were classified if there were solitarious or no nymphs identified. This approach is similar to other studies analyzing desert locust reproduction successes (Piou et al. 2017, Kayalto et al. 2020).

\section{From points to gridded data}

We centered all data points within either a $5 \times 5 \mathrm{~km}$ grid for desert locusts or a $1 \times 1 \mathrm{~km}$ grid for Australian plague locusts and collected data from that entire area to reflect nymph mobility, gregarization and scale of NDVI importance for population outbreaks. The desert locust is considerably larger than the Australian plague locust and nymphs can walk up to 7-12 km (Ellis and Ashall 1957, Coppen 1999) whereas
Australian plague locusts can walk a maximum of $2 \mathrm{~km}$ from the hatching site before becoming adults (Hunter et al. 2008). Given that gregarization happens at local scales (Collett et al. 1998) and the importance of local habitat conditions on nymph growth (Veran et al. 2015), the grid sizes are appropriate trade-offs. Lastly, whereas spatial scale of importance for NDVI resolution has not been reported for Australian plague locust, Piou et al. (2013) found that averaged NDVI of $0.25 \times 0.25 \mathrm{~km}$ and $0.75 \times 0.75 \mathrm{~km}$ were not as good of predictors as $1.25 \times 1.25 \mathrm{~km}$ and that vegetation quantity (e.g. number of NDVI pixels above 0.14) is an important predictor for presence/absence of desert locust at a scale of 8 $\times 8 \mathrm{~km}$. Given these factors and understanding that nymph mobility varies widely with landscape structure which varies largely between the Australian, African and southwest Asian drylands (Maestre et al. 2021), we selected $5 \times 5 \mathrm{~km}$ grid for desert locusts and $1 \times 1 \mathrm{~km}$ grids for Australian plague locusts. Grid size selection should be based on current literature and future research endeavors should consider carefully what grid size is used as there is not one correct size.

\section{Biological relevant spatial level sourcing}

All levels (zone, land unit and season) were selected to best reflect the spatiotemporal variation within each species range (Table 1). For the Australian plague locust, we used the major rainfall classification zones as the geographic region level which are six large climatic zones based on seasonal rainfall distribution (<www.bom.gov.au>) (Fig. 1B; Supporting information). For land unit, we used the Interim Biogeographic Regionalisation for Australia (IBRA) ver. 7 dataset (<www.environment.gov.au/land/nrs/science/ibra $>$ ) (Fig. 1C; Supporting information). For seasonality, there are few records from winter months because the bulk of the population are in dormant eggs stages, as such we left winter out of the models. Seasons were separated by the following dates:

- Spring: 2 September-1 December

- Summer: 2 December-29 February

- Autumn: 1 March-1 June

- Winter: 2 June-1 September

For the desert locust, we used the outbreak area model as suggested by Uvarov (1928) (Fig. 1F; Supporting information) for the geographic region level. There are two categories at this level which are recession and invasion zones. These zones represent desert locust ecology where they persist throughout generations in the recession zone and periodically swarm and outbreak in the invasion zones. For the land unit level, we used the World Wildlife Fund's Terrestrial Ecoregions of the World (Fig. 1G; Supporting information) (Olson et al. 2001). The bioregions and ecoregions used for each species differ slightly. The Australian bioregions were constructed with vegetation characteristics in mind. The World Wildlife Fund's Ecoregions are constructed from similar soil characteristics and topography and have a more generalized approach to acknowledging major vegetation structure. To ensure that smaller levels were assigned one single category 
Table 1. Variables used in this study with structure and source.

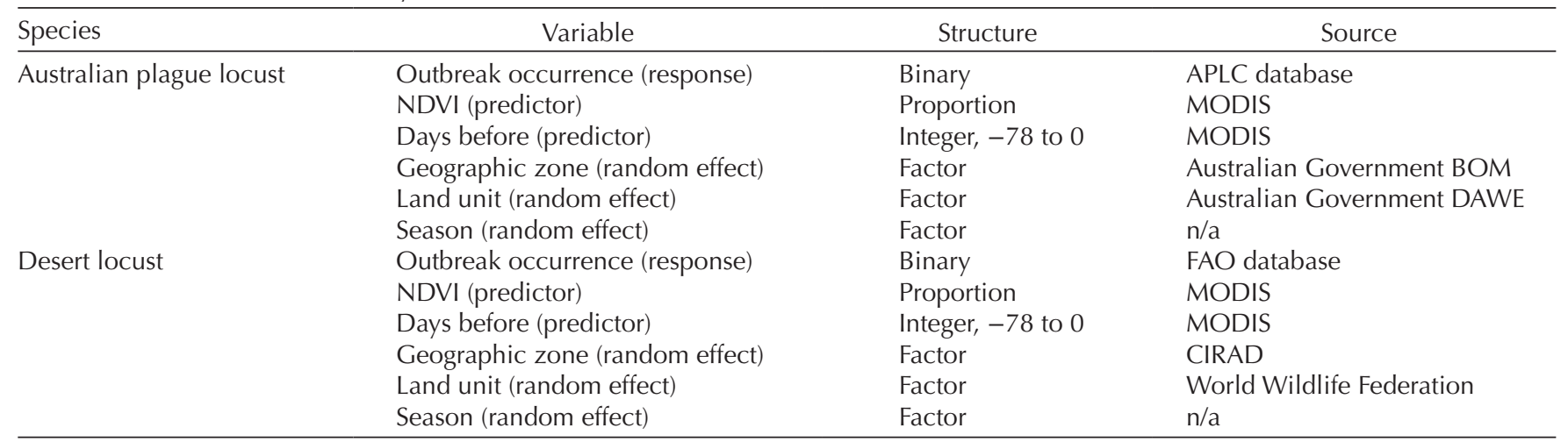

for each higher level, we assigned each observation grid and land unit the category in which it overlapped the most with.

Since the species range encompasses a large geographic area, traditional seasons are not applicable in this situation. Instead, we used a temporal grouping that reflected large scale migration trends of this species (Symmons and Cressman 2001) (Fig. 1F, Supporting information). This led to the following seasonal division:

- Spring: 1 April-30 June

- Summer: 1 July-30 September

- Autumn: 1 October-31 December

- Winter: 1 January-31 March

\section{Remotely sensed data preparation}

The normalized difference vegetation index (NDVI, Pettorelli 2013) is an established method for estimating vegetation amount and condition from multispectral scanners. The index is calculated as a ratio of the contrast between the surface reflectance infra- (NIR) and visible-red (Red) wavelength and the sum of these two bands (NIR - Red/NIR + Red), which indicates the amount of actively photosynthetic material covering the pixel. We used 8-day averaged MODIS (Terra Surface Reflectance 8-Day Global 250 m, MOD09Q1 v006) imagery captured between 2000 and 2019, collected from Google Earth Engine (Gorelick et al. 2017). This allowed us to account for variances in imagery based on cloud coverage, low view angle, aerosols and other potential image collection biases. Because locust plagues do not occur in and rarely go into urban areas, we did not need to exclude urban areas. In general, in the habitat of both species, high values of NDVI represent food plants and when averaged into ecoregions/ bioregions, this land cover type only represents a negligible proportion of the total area.

We used the fixed $1 \times 1 \mathrm{~km}$ and $5 \times 5 \mathrm{~km}$ grids established for the locust data to resample the $0.25 \times 0.25 \mathrm{~km}$ pixels from sequential MODIS composite images. For all observation grids, we sampled 10 prior, 8-day interval images and calculated the mean and standard deviation for all pixels within or touching each grid cell. The total number of pixels in each cell varied slightly over time because of image navigation and registration. Composite image median dates were used to calculate the number of days prior to the locust observation. Since image overpass and locust observation dates did not frequently align, the 10th prior image was not always exactly 80 days before. The main fixed effects used in all models are the NDVI value and days before locust observation.

\section{Model construction}

To understand the hierarchical relationship between land units, geographic zones and seasons, we used a combination of hierarchical models and generalized additive models (GAM), known as hierarchical generalized additive models (Wood 2017, Pedersen et al. 2019). Briefly, GAMs allow the relationship between the dependent and independent variables to be described by smoothing curves (e.g. splines). These curves allow for the estimation of non-linear trends whereas traditional linear models can only at best estimate polynomial relationships. The flexibility of GAMs allows for an easy extension into hierarchical modeling where smoothed relationships can vary between groups (Pedersen et al. 2019). In our study, we fitted hierarchical GAMs (family: binomial, link: logit) to nymph density data with the variables listed in Table 1 . We constructed two dimensional smoothers (Wood et al. 2016) for both NDVI values and the number of days prior to locust observation dates and latitude and longitude. This allowed us to create probability heat maps from the modeled results (Fig. 3B). The inclusion of latitude and longitude allowed us to account for spatial autocorrelation (Clayton et al. 1993) in the locust survey datasets that, if left unaccounted, would bias the results for both Australian plague locust (Moran's I: observed: 0.18 , expected: 0 , p-value: $<0.001)$ and desert locust (Moran's I: observed: 0.06, expected: 0, p-value: < 0.001 ). We ensured spatial autocorrelation was low by mapping residuals for each species (Supporting information).

\section{Model validation and selection}

Overall, models were first validated using diagnostic Q-Q plots. Basis dimensions were insured large enough to capture nonlinear trends while accounting for computational time (Wood 2017; Supporting information) before models were selected. We used null space penalization to test 


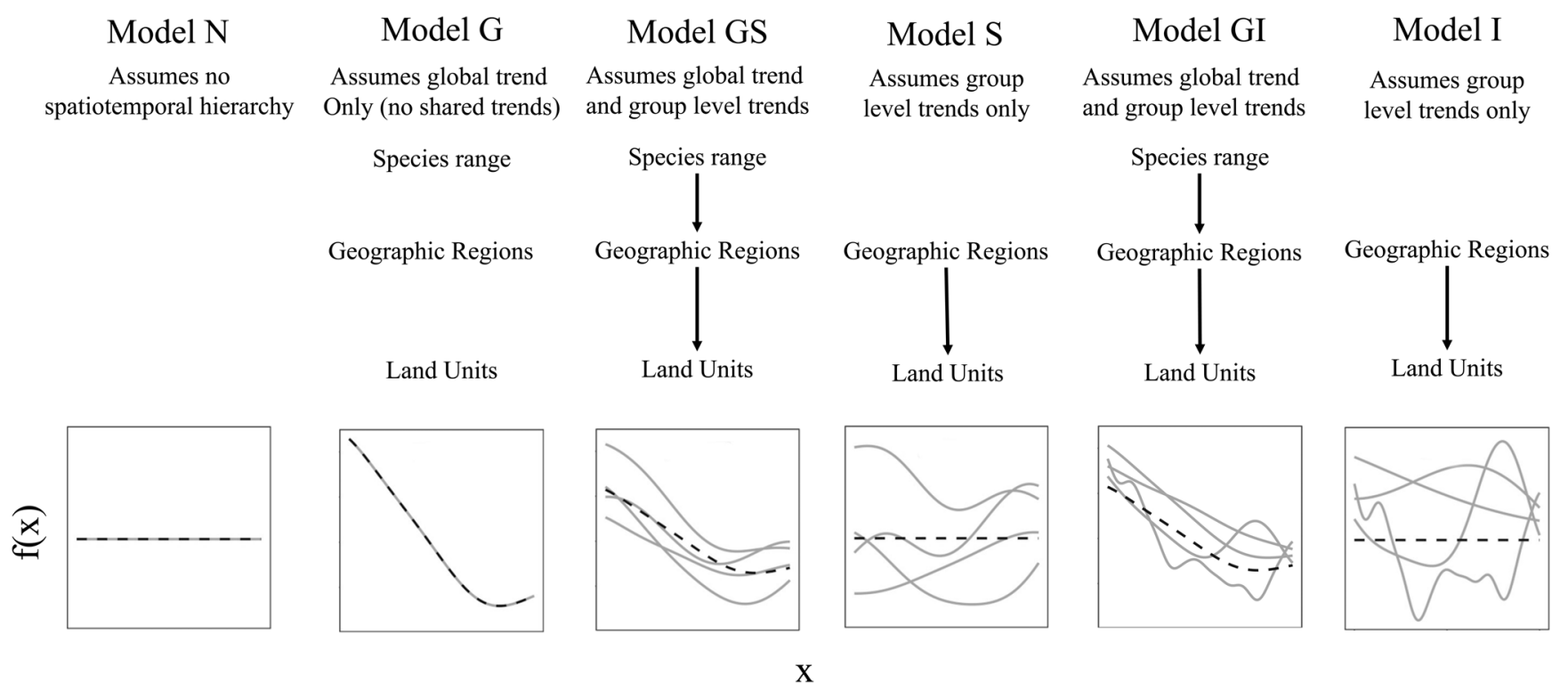

Figure 2. Hierarchical model structure used to test the spatiotemporal nature of locust outbreaks. Top: shows the varying hierarchical leveling between model design. Bottom figures graphically show the differing model design. Black dashed lines represent global trend and grey solid line represents group level trends. The model GS and S have similar smoothed group level trends (all group-level trends have a shared penalty) whereas model GI and I have different group level trends (group-level trends have individual penalties). Bottom figures are from Pedersen et al. (2019) and are under Creative Commons 3.0 license.

whether there were nonlinear or linear trends in the model (Marra and Wood 2011, Wood 2017). Since there can be discrepancies between different model selection criteria (Pedersen et al. 2019), we reported four metrics: Akaike information criterion (AIC), Bayesian information criterion (BIC), the deviance from an out-of-sample (OOS) dataset (e.g. cross-validation) termed OOS deviance as discussed by Pedersen et al. (2019), and area under the receiver operating characteristic curve (AUC). For OOS deviance, we built models using $70 \%$ of the dataset, termed the training set. We then quantified predicted deviance from known outbreak and non-outbreak data using the remaining 30\% of the dataset, termed the validation set. This deviance can be interpreted similarly as residual sum of squares for a linear regression with lower values meaning a better fit model (Wood 2017, Pedersen et al. 2019). Receiver operating characteristic curves (ROC) for the final models are shown in Supporting information.

\section{Hierarchical structure}

To confirm the levels selected (geographic zone, land unit and season) were biologically relevant, we constructed models with all combinations of the three levels and selected as discussed above (Model validation and selection section). Since we were not yet concerned about spatiotemporal hierarchy, we constructed all models under the same framework (model G; Fig. 2) and used only $70 \%$ of the data to increase computational speed. Therefore, it is not advisable to compare selection criteria values between these models and the final hierarchical model testing (Supporting information).
We tested the validity of our hypothesized spatial structure (species range $>$ geographic zone $>$ land unit) after confirming that including all biologically relevant levels performed better than any subset. To do this, we constructed six different models with varying structures including all biological levels following the protocol of Pedersen et al. (2019). Briefly, we constructed a model with no hierarchy (model $\mathrm{N}$ ), a model with only a global level (model G), a model with global and similarly smoothed (e.g. all groups share a common penalty) group level trends (model GS), a model with similarly smoothed group level trends only (model S), a model with global level and differently smoothed group level trends (model GI, e.g. all groups have an independent penalty), and a model with only differently smooth group level trends (model I). Due to the nested relationship of model GS and model S, specific interactions between geographic zones and land units cannot be assessed via p-values. For models GI and I, specific interactive terms can be assessed. Models are visually explained further in Fig. 2. Once all models were constructed, we selected the models with AIC, BIC, OOS and AUC criteria as discussed in Model validation and selection section.

\section{Statistical and GIS software used}

Remotely sensed imagery collection and preparation were done using QGIS (Team 2020) and in the python environment with GeoPandas (Jordahl 2014). Statistics were conducted in $\mathrm{R}$ (<www.r-project.org >) within the tidyverse framework (Wickham 2018). All models were constructed and selected using the mgcv package (Wood 2017) and validated with the gratia package (Simpson 2019). 


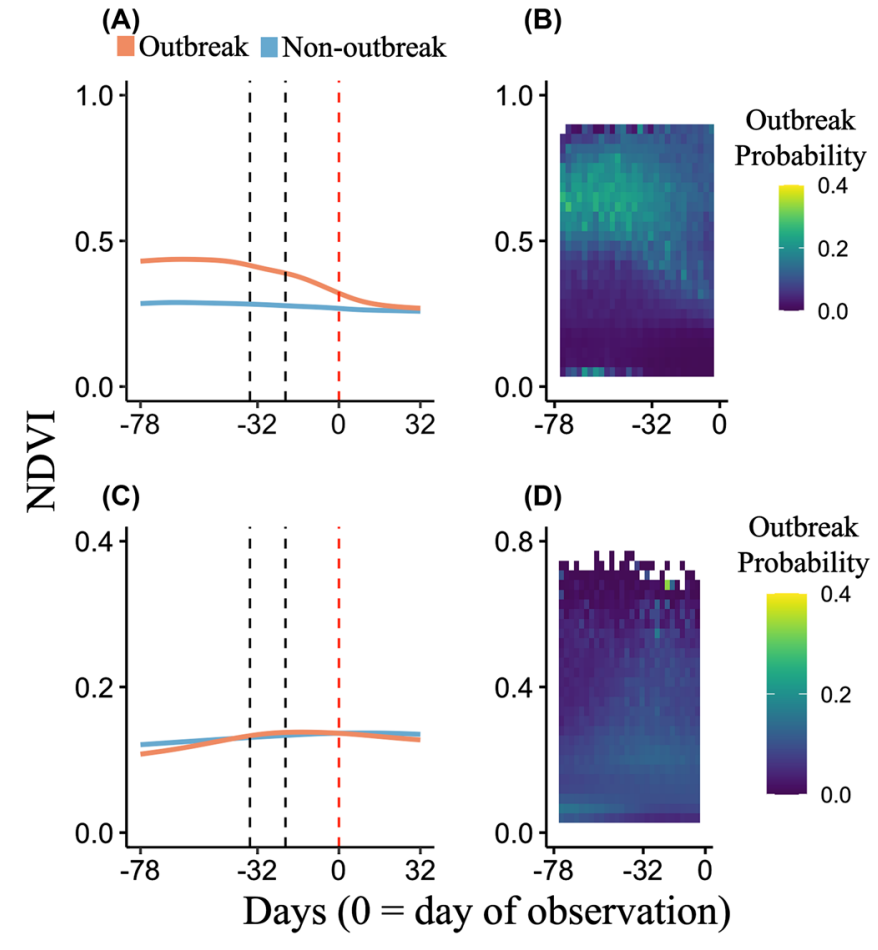

Figure 3. Global pattern for the Australian plague locusts (A), (B) which are characterized by long preceding vegetation growth between 78 and 32 days before outbreaks and desert locust (C), (D) which are characterized with vegetation pulses 32-20 days before outbreaks. Left: NDVI trends for outbreak and non-outbreak observations over time. Vertical red line is the observation date, black lines represent hypothesized nymph hatching date. Right: Modeled outbreak probability for preceding NDVI. We did not include succeeding NDVI values into models.

\section{Results}

\section{Biologically relevant level selection}

For both species, each of the selection criteria yielded models that included all geographic scales and season, although there were ties with models that left out some levels (Supporting information). For the desert locust, AIC, BIC, OOS deviance selected to include all levels: geographic zone, land unit and season while AUC selected for two models: all levels and geographic zone and land unit only models (Supporting information). For the Australian plague locust, AIC, BIC, OOS and AUC selected to include all levels.

\section{Overall hierarchy selection}

Overwhelmingly, all criteria selected either model GS (global and group level) or model S (group level trends with no global trend) for both species (Supporting information). This indicates that regardless of the relationship between preceding vegetation growth and outbreaks, the responses of both species were spatially structured in that the inclusion of zone and land units increased model fit. However, model selection results differed between model GS and model $S$ for both species
(Supporting information). For the Australian plague locust, $\mathrm{AIC}$ and BIC selected for model GS (Supporting information) whereas OOS selected model S and AUC was tied between the two models. For the desert locust, AIC and OOS both selected model S, whereas BIC selected model GS and AUC was tied between model $S$ and model GS (Supporting information). OOS deviance values for each level are given in the Supporting information. The model summaries for model GS and model $S$ for both species can be seen in Table 2. Summaries for the four other models can be seen in Supporting information.

\section{Species range view}

At the overall species range level, nymph outbreaks were distinct from non-outbreaks in terms of preceding NDVI values resulting from vegetation growth (Fig. 3, Table 2, Supporting information). However, the two species show clearly differing responses. Australian plague locust outbreaks are preceded by vegetation growth between 78 and 32 days before the observation (Fig. 3A-B). On the other hand, desert locust outbreaks were characterized by weaker albeit significant vegetation pulses between 32 and 20 days before the observation (Fig. 3C-D).

\section{Geographic region view}

Outbreak response to preceding vegetation growth varied between geographic regions (Fig. 4, 5, Table 2, Supporting information). For the Australian plague locust, vegetation pulses up to 32 days prior were associated with summer outbreaks in summer-dominant rainfall geographic zones (Fig. 4A-C), whereas outbreaks in all other zones required longer preceding vegetation growth (Fig. 4D-E). For the desert locust, outbreaks in both invasion and recession zones were characterized by short prior vegetation pulses (Fig. 5, Table 2, Supporting information). However, NDVI values before outbreaks were higher in the invasion zone than in the recession zone (Fig. 5) Further, vegetation in the invasion zone was drier in outbreak episodes compared to non-outbreaks (Fig. 5A).

\section{Land unit and season levels}

Due to the large number of land units separated into three or four seasons each, we are unable to explicitly report on all findings. However, each land unit is given a summary page within the Supporting information with both trend lines (Fig. 3A) and modeled results for model GS and S (Fig. 3B) reported (Supporting information). There are notable differences in outbreak response to vegetation both between and seasonally within land units. Regardless of these differences, there are two overarching patterns: long preceding vegetation growth and pulses in vegetation growth (for selected examples see Fig. 6).

\section{Discussion}

We modeled the incidence of locust outbreaks for two species (Australian plague locust, Chortoicetes terminifera and desert 
locust, Schistocerca gregaria) using hierarchical generalized additive models (GAMs) driven by NDVI as an indicator of herbaceous vegetation productivity. Our study uses the hierarchical patch dynamics (HPD) framework to account for the spatiotemporal variation of locust swarms and demonstrates that model performance is improved by the integration of biologically relevant scales. This is shown in two taxonomically distinct locust species where the relationship between preceding vegetation growth and outbreaks varies spatiotemporally (Fig. 3, 4, 5). However, regardless of their inherent ecological differences, adding biologically relevant spatiotemporal hierarchy greatly improved model fit in both species (Supporting information). This suggests that when making large scale conclusions about the ecological causes of locust swarms, and likely other mobile animals, spatial hierarchy should be considered. The relationship between preceding vegetation growth and locust outbreaks likely varies between these species due to the large climatological differences between their respective ranges in Africa, Southwest Asia and Australia and their distinct ecologies reflecting their adaptations to those differences. Further modeling could assess other important variables such as temperature and nutrition which have impacts on the organismal to landscape levels for locusts.

For locust management, our study reinforces the importance of prior rainfall-induced vegetation growth on the likelihood of outbreaks. The results support the current strategies of using plant productivity measures such as NDVI to direct surveys and increase the probability of detecting nymph outbreaks to allow for possible proactive intervention (Pedgley 1973, Cressman 2008, 2013, Deveson 2013). Further, it highlights the spatiotemporal structuring of outbreaks which is important for forecasting efforts globally. For example, locust managers can use the land unit summary figures (Supporting information) to help decide where and when to send surveyors throughout the locust season. Surveying for locusts is costly due to the wide area of potential habitat and the limited resources available. Our study provides a helpful aid to better inform and increase the effectiveness of these crucial surveys. Further, our use of HPD and hierarchical generalized additive models allows for spatiotemporal variation to be directly modeled and identified. Therefore, the methods and findings provide a useful tool in understanding and predicting the spatiotemporal variation of population dynamics broadly.

\section{Preceding vegetation growth and locust outbreaks}

It is well documented that preceding vegetation growth is an important predictor of acridid population dynamics (Chapman and Joern 1990). The environments of both species are arid and semi-arid lands characterized by the resource pulse paradigm, meaning that primary productivity and herbivore response is driven largely by variable rainfall events (Noy-Meir 1973, 1974). In these habitats in dry conditions, nymph populations will be unable to develop into adults, which is the breeding and migratory stage. However, few 


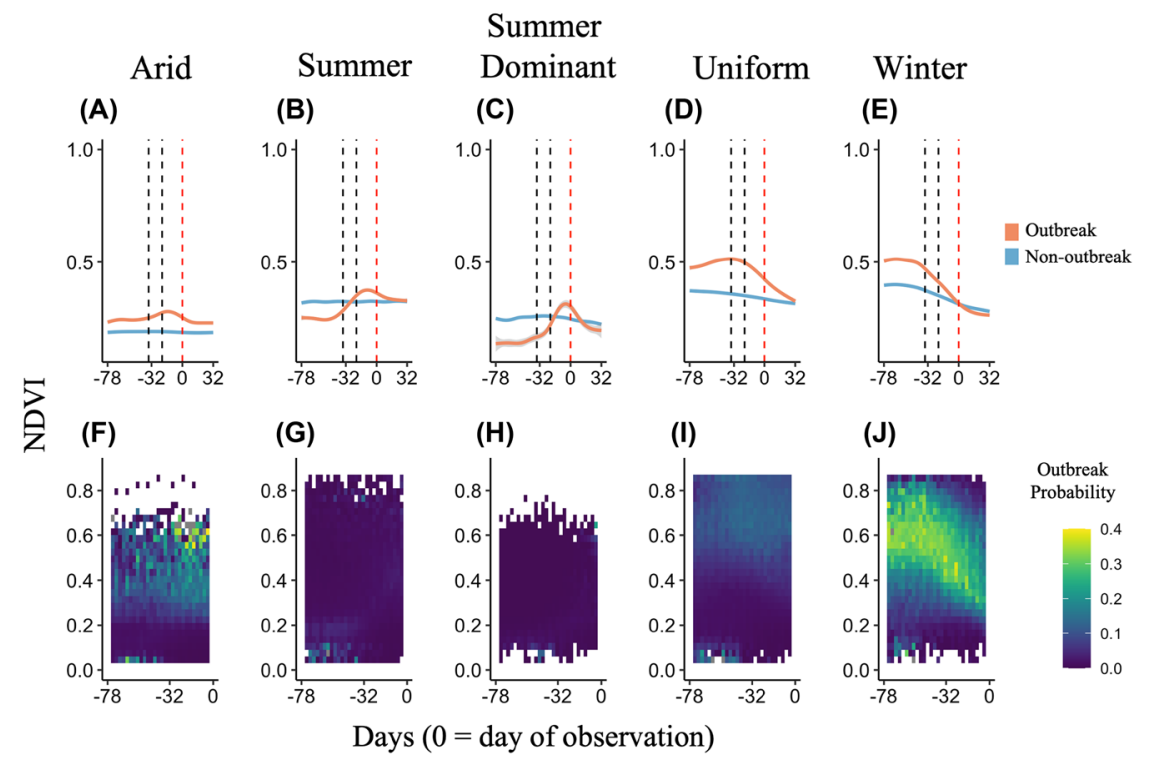

Figure 4. Australian plague locust geographic region pattern for raw (A)-(E) and modeled probability (F)- $(J)$ for the relationship between preceding vegetation growth and outbreaks. Two patterns emerged: long preceding vegetation growth in uniform and winter geographic regions $(\mathrm{D}, \mathrm{E}, \mathrm{I}, \mathrm{J})$ and vegetation pulses in arid summer and summer dominant regions (A-C, F-H). Top: raw NDVI trends for outbreak and nonoutbreak observations over time. Vertical red line is the observation date, black lines represent hypothesized nymph hatching date. Bottom: Modeled outbreak probability for preceding NDVI. We did not include succeeding NDVI values into models.

researchers have statistically investigated the daily temporal dynamics of this relationship. Our study generalizes the findings of previous studies that used NDVI binned or averaged into discrete time ranges in both species (Deveson 2013,
Piou et al. 2013). Additionally, we show there are important differences between preceding vegetation growth and outbreak responses at different spatiotemporal levels which were not accounted for statistically in previous studies.

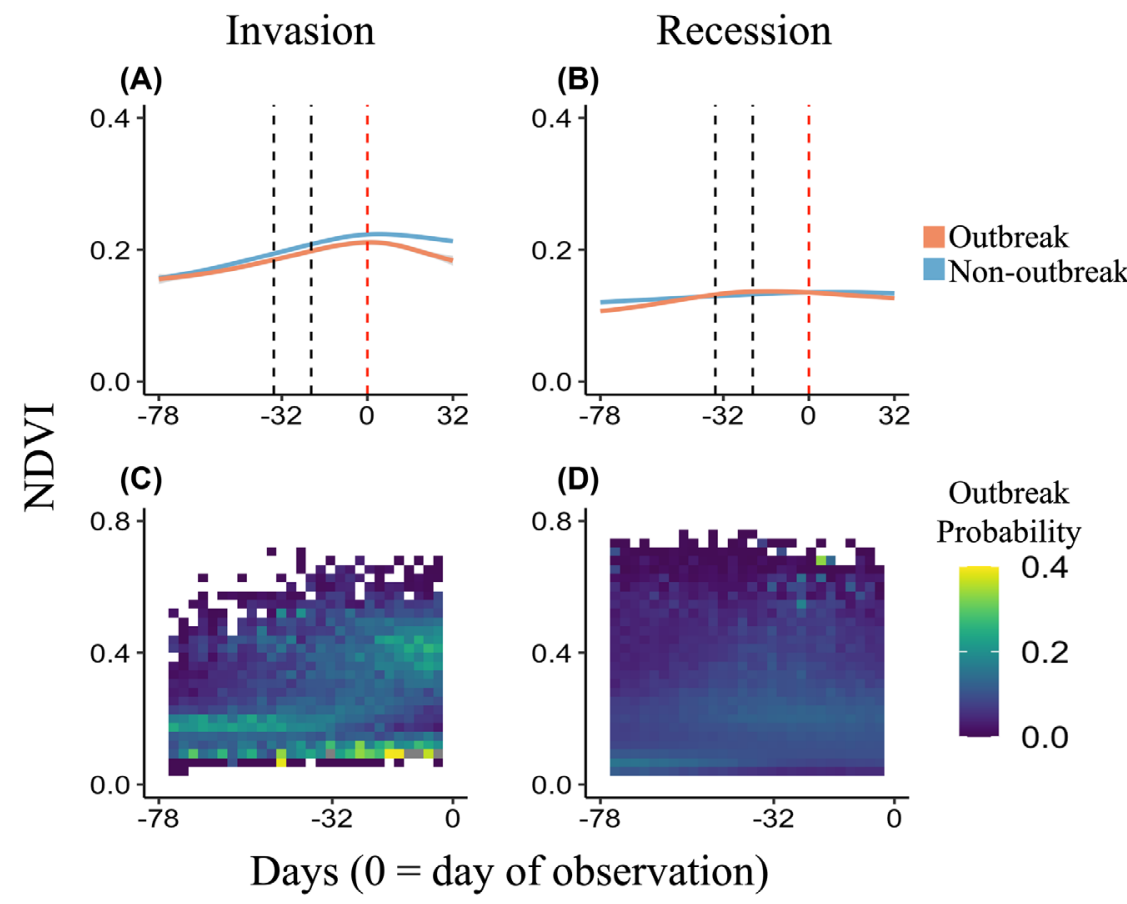

Figure 5. Desert locust geographic region level pattern for raw (A), (B) and modeled probability (C), (D) for the relationship between preceding vegetation growth and outbreaks. Both regions are characterized by vegetation pulses. Top: raw NDVI trends for outbreak and nonoutbreak observations over time. Red line is the observation date, black lines represent hypothesized nymph hatching date. Bottom: Modeled outbreak probability for preceding NDVI. We did not include succeeding NDVI values into models. 
Australian plague Locust

(A) Channel Country

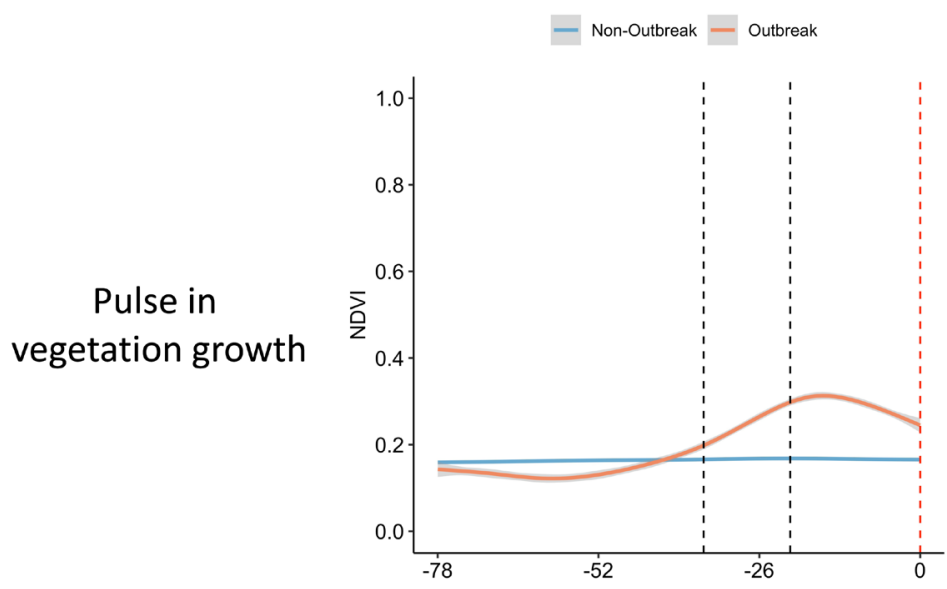

(C) Murray Darling Depression

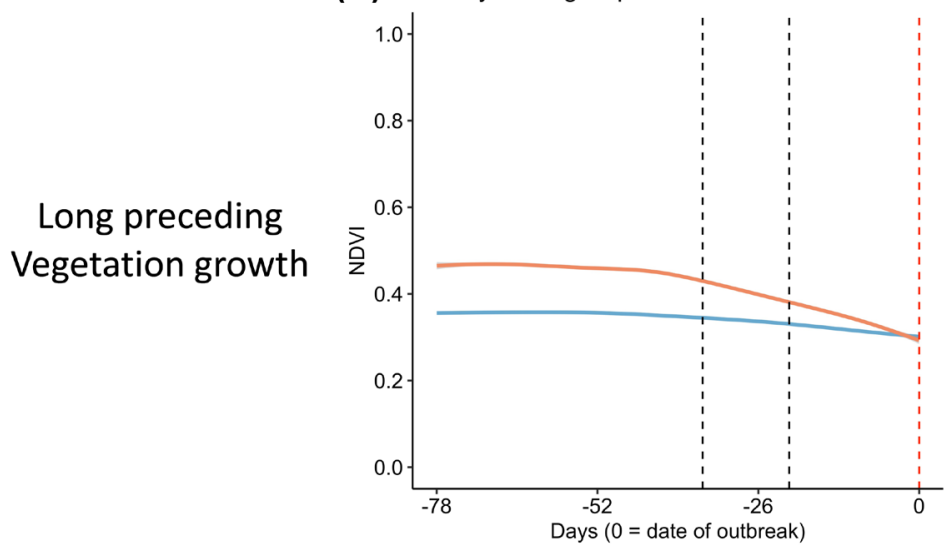

Desert locust

(B) Ethiopian xeric grasslands and shrublands

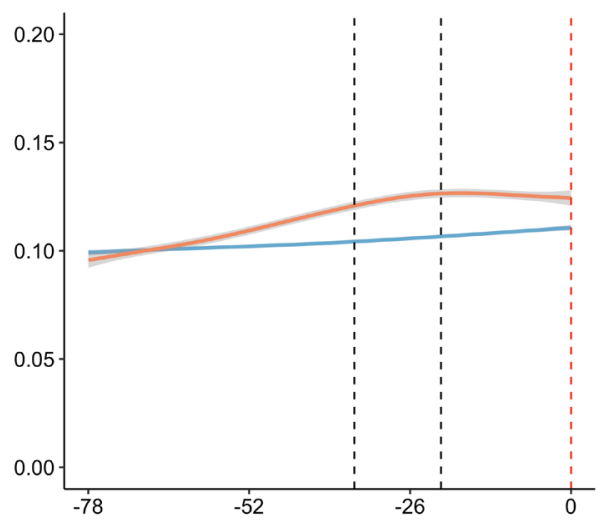

(D) South Iran Nubo-Sindian desert and semi-desert

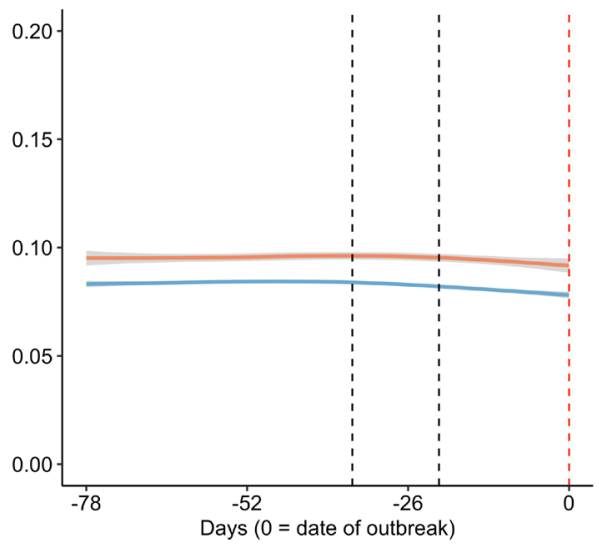

Figure 6. Four land unit examples for Australian plague locust (A), (C) and desert locust (B), (D) relationship between preceding vegetation growth and outbreaks. Both species are characterized largely by either pulses (A, B) or long preceding (C, D) vegetation growth trends depending on land unit. Red line is the observation date, black lines represent hypothesized nymph hatching date.

\section{Spatiotemporal variation between preceding vegetation and} outbreaks

Regardless of species and spatiotemporal level, two major patterns between vegetation growth and outbreaks emerged: long preceding vegetation growth (> 32 days before) and vegetation pulses ( -32 days before). The vegetation pulses occurred around the time of the hypothesized hatching date for the nymph outbreak which is two to three weeks before outbreak observation (Fig. 3). This is supported by model criteria selecting model GS and S (similar smoothed relationships between levels) over model GI and I (differently smoothed relationships between levels) (Fig. 2, Supporting information). Most of the desert locust models at all levels had vegetation pulses whereas the Australian plague locust levels had both patterns. These patterns are a product of climatic differences between the habitat and each species' ecology. The majority of the desert locust habitat is hyper-arid and hot and given the wide geographic distribution, winter does not halt population growth (Uvarov 1977, Ceccato et al. 2007). As such, responses to brief vegetation pulses are therefore prominent. The Australian plague locust's environment has on average a higher mean annual precipitation and experiences a cold winter which halts population growth for part of the year (Wardhaugh 1986, Hunter et al. 2001). Long preceding vegetation growth is associated with bioregions within the winter and uniform dominant rainfall zones which is in the southern half of the species range (Supporting information). This indicates that late winter and early spring temperature and rainfall-induced vegetation growth is important for nymph survival. Within the summer and arid rainfall zones in the northern half of the species range, the shorter preceding vegetation pulse is prominent (Fig. 4). Outbreaks in these areas happen later in the season and vegetation growth following rainfall is needed for successful breeding.

While we did not explicitly model adult migration and behavioral ecology, it is important to recognize that the presence of nymphs indicates prior adult oviposition choices. Without egg laying in the localized area, there would be no nymphs to consume the available vegetation. Both species conduct wind-assisted migrations which increases the chances of breeding in habitat areas with recent rain (Dingle 
2014). There are migratory differences between species based on large-scale climatic patterning. It is important to note that these migratory trends for both species are generalized. It is common for both species to migrate in response to disturbed weather associated with regional weather systems rather than prevailing wind patterns (e.g. trade winds and westerlies). Often, but not always, such systems are associated with an increased rainfall probability. Given the erratic nature of rainfall in semi-arid regions, locusts are believed to be adapted to use these systems to transport them to areas of potentially more favorable vegetation growth conditions with accompanying increased egg and nymph survivorship chances (Farrow 1990). Our modeling demonstrates episodes where rainfall, adult migration and vegetation growth all occur at the appropriate timing to produce a nymph outbreak.

For the Australian plague locust, any migration events southward in autumn (into winter dominated rainfall regions) and northward in late spring (into summer dominated rain regions) have increased probability of successful breeding (Clark 1970, 1971, Farrow 1979, Deveson and Walker 2005, Deveson et al. 2005). After southward migrations in autumn, most laid eggs go into diapause (a period of suspended development) due to the onset of winter and colder temperatures, which delays hatching until spring. Native temperate-climate grasses remain green throughout winter and into spring due to lower temperature and decreased evaporation which explains the long preceding vegetation growth prior to outbreaks in these areas (Fig. 4D-E). Northward migrations relocate locusts into the summer dominant rainfall zones in early summer which corresponds with the commencement of the wet season. Therefore, any nymphal outbreaks in this region are preceded by vegetation pulse (Fig. $4 \mathrm{~A}-\mathrm{C}$ ).

For the desert locust there are two large-scale migration trends in West Africa and the Red Sea area. In West Africa, migrations usually follow the inter-tropical convergence zone (ITCZ) with southward in winter/spring and northward displacements in summer (Rainey 1963, Pedgley 1980). In the Red Sea area, winter migrations from the Middle East occur to the Indo-Pakistan and the Red Sea's western shores areas. Summer migrations then head back towards the Middle East. Overcoming cooler temperatures for desert locusts is not as important as compared to Australian plague locusts due to being in a hotter climate. The important factors for outbreaks to occur is recent rainfall and vegetation pulses. Therefore, most of the desert locust hierarchical levels demonstrated the vegetation pulse pattern. While both the invasion and recession zone demonstrate this pulse, outbreaks in the invasion zone on average were greener than those in the recession zone (Fig. 5). This is partly because the invasion zone has a higher mean annual precipitation than the recession zone.

Trajectory migratory modeling of pests like locusts is a large field of study (Parry 2013) and there is great potential to couple our findings with this type of modeling. We hope that this study spurs interest in coupled trajectory and habitat quality indices (like NDVI) modeling of insect outbreaks.

The smaller deviations seen between all spatiotemporal levels are likely the result of other environmental variables in particular vegetation. Vegetation communities differ considerably in their composition among the Australian bioregions (Thackway and Cresswell 1995) and among the African ecoregions (Olson et al. 2001) considered in this study. Thus, the occurrence, extent and intensity of outbreaks will be influenced by the unique composition of plant communities within the specific land unit, and how these communities are distributed to the changes in available soil composition, nutrients, temperature and precipitation (Van der Werf et al. 2005, van Huis 2007, Hassler et al. 2010, Mao et al. 2014). These deviations could lead to interesting hypotheses about the spatiotemporal population dynamics at higher trophic levels. Importantly, populations in our study responded differently to the same precipitation regime in different regions due to vegetation productivity being spatially variable.

We show here that if a spatial hierarchy is not modeled (e.g. model N) important trends are masked. As such, when we include spatial hierarchy in the modeling process, overall fit is improved. When modeling large scale animal population dynamics, it is important to not only identify the biologically relevant spatiotemporal hierarchy of the system but also include the hierarchy in statistical model building. Interestingly, model selection criteria identified model $S$ (without global level) for desert locusts and model GS (with global level) for Australian plague locusts. Since the desert locust species range encompasses a considerable geographic area with enormous abiotic/biotic variation and winter does not halt production, it makes sense that there would not be a common trend shared between the zones and land units. For Australian plague locust outbreaks to occur, there must be long preceding vegetation growth in the winter rainfall dominated areas since each locust outbreak season is suspended during the winter months with the onset of colder temperatures. Therefore, the global level pattern of this species is one of long preceding vegetation growth and model selection criteria selected for model GS.

\section{Additional environmental factors and hierarchical levels for outbreaks}

There are a plethora of factors that influence population dynamics in addition to the ones mentioned in the section above (Spatiotemporal variation between preceding vegetation and outbreaks section). We did not include these factors because this would likely introduce concurvity - the nonparametric equivalent of multicollinearity that in GAMs can lead to variation in the effects of independent variables being underestimated (Morlini 2006) plus overcomplicate the models. One important variable to consider is annual treatments (e.g. pesticide spraying) for both species which likely influences the yearly population dynamics seen. Whereas NDVI is widely used for vegetation monitoring and mapping there are other indices that may be better for areas with sparse vegetation like precipitation. Going into the future, including scalerelevant measures of these variables would increase model fit and provide insight into other factors that induce locust swarms. Due to the nature of these datasets, we were unable to recognize smaller hierarchical levels such as within-land unit 
abiotic/biotic variation, microhabitats and smaller temporal scales (e.g. days and weeks). Within land unit there could be differences between populations (Mangeon et al. 2020) and microhabitats. For microhabitats, temperature and soil moisture likely play an important role in regulating individual locust performance and work is currently underway at this scale (Kearney and Porter 2009, Kearney et al. 2014).

\section{Hierarchical patch dynamics: integrating ecology on multiple scales}

The hierarchical patch dynamics paradigm is a way to account for emergent properties that arise because of interactions at and between different scales (Newman et al. 2019). In this study, we show that breaking down species range trends into discrete biologically relevant levels reveals patterns and processes that are not apparent at other scales. If this spatiotemporal variation is not accounted for, species population dynamics modeling will be limited. Spatiotemporal variation is clearly an important aspect for locust swarms, and this is also likely the case for a broad range of animals. There is a need for explicitly integrating ecological phenomena across multiple scales (Carpenter and Turner 2017). Hierarchical generalized additive models allow for information to be shared between levels (Pedersen et al. 2019) and could increase both the theoretical and practical applications of the HPD theory. This approach provides a useful tool for integrating ecology at multiple scales. We hope this study spurs further research in explicitly accounting for spatiotemporal variation within population dynamics.

Acknowledgements - We thank Chris Adriaansen and the APLC staff for the support throughout from origin to writing this article. Further, we thank Gavin Melville, Marja Simpson and Kim Broadfoot for their assistance with the statistics and geoprocessing of datasets. We thank both the Orange Agricultural Institute and the Dubbo Regional Office of the NSW Dept of Primary Industries for logistical support. We thank Research Computing at Arizona State University for providing resources that have contributed to the research results reported within this article (<www. researchcomputing.asu.edu $>$ ). We thank the Stacks Exchange and Overflow communities for model building assistance.

Funding - This work was supported by the Australian Plague Locust Commission, Arizona State Univ., School of Life Sciences RTI grant and SIRG Travel Award, Australian Rangeland Society, Orthopterists' Society and the Foundation for Food and Agricultural Research's New Innovator Award [593561 to AC]. C. Piou was funded by ANR-JCJC PEPPER (ANR-18-CE32-0010-01).

Conflict of interest - The authors declare no conflicts of interests.

\section{Author contributions}

Douglas Lawton: Conceptualization (lead); Formal analysis (lead); Funding acquisition (lead); Investigation (lead); Methodology (lead); Resources (equal); Software (equal); Writing - original draft (lead). Peter Scarth: Data curation (supporting); Methodology (equal); Resources (lead); Software (equal); Writing - review and editing (supporting). Edward Deveson: Conceptualization (supporting);
Data curation (equal); Investigation (equal); Supervision (equal); Writing - review and editing (equal). Cyril Piou: Data curation (equal); Investigation (equal); Methodology (equal); Supervision (equal); Writing - review and editing (equal). Allan Spessa: Methodology (equal); Supervision (equal); Writing - review and editing (equal). Cathy Waters: Conceptualization (equal); Funding acquisition (equal); Investigation (equal); Methodology (equal); Resources (equal); Supervision (equal); Writing - review and editing (equal). Arianne Cease: Conceptualization (equal); Funding acquisition (equal); Methodology (equal); Project administration (equal); Resources (equal); Supervision (equal); Validation (equal); Writing - review and editing (equal).

\section{Transparent Peer Review}

The peer review history for this article is available at $<$ https:// publons.com/publon/10.1111/ecog.05763>.

\section{Data availability statement}

Desert locust data is publicly available via the FAO locust hub: <https://locust-hub-hqfao.hub.arcgis.com>. As per the Australian Plague Locust Commission guidelines, the dataset cannot be made publicly available as it requires specific explanation to understand some aspects of its contents. Those who are interested in these data should contact the APLC director who, at the time of the publication, is Chris Adriaansen.

\section{Supporting information}

Any supporting information associated with this article is available from the online version.

\section{References}

Andrewartha, H. G. and Birch, L. C. 1954. The distribution and abundance of animals. - Univ. of Chicago Press.

Barbosa, P. et al. 2012. Insect outbreaks revisited. - Wiley.

Carpenter, S. R. and Turner, M. G. 2017. Twenty years of ecosystems: emerging questions and challenges. - Ecosystems 20: 2016-2018.

Ceccato, P. et al. 2007. The desert locust upsurge in West Africa (2003-2005): information on the desert locust early warning system and the prospects for seasonal climate forecasting. - Int. J. Pest Manage. 53: 7-13.

Chapman, R. F. and Joern, A. 1990. Biology of grasshoppers. Wiley.

Clark, D. P. 1970. Plague dynamics of the Australian plague locust, Chortoicetes terminifera (Walk.). - In: In: Hemming, C. F. and Hemming, T. H. C. (eds), Proceedings of the International Study Conference on the Current and Future Problems of Acridology, 1970, pp. 275-287. Centre for Overseas Pest Research, London, UK.

Clark, D. P. 1971. Flights after sunset by the Australian plague locust, Chortoicetes terminifera (Walk.) and their significance in dispersal and migration. - Aust. J. Zool. 19: 159-176. 
Clayton, D. G. et al. 1993. Spatial correlation in ecological analysis. - Int. J. Epidemiol. 22: 1193-1202.

Coe, P. K. et al. 2011. Validation of elk resource selection models with spatially independent data. - J. Wildl. Manage. 75: 159-170.

Collett, M. et al. 1998. Spatial scales of desert locust gregarization. - Proc. Natl Acad. Sci. USA 95: 13052-13055.

Condit, R. et al. 2000. Spatial patterns in the distribution of tropical tree species. - Science 288: 1414-1418.

Coppen, G. D. A. 1999. A simple model to estimate the optimal separation and swath width of ULV-sprayed barriers of Chitin synthesis inhibitors (CSI) to control locust hopper bands. Crop Protect. 18: 151-158.

Council, N. R. 2001. Grand challenges in environmental sciences. - National Academies Press.

Cressman, K. 2001. Information and forecasting. Desert locust guidelines. - Food and Agricultural Organization of the United Nations.

Cressman, K. 2008. The use of new technologies in desert locust early warning. Outlooks on pest management. - Research Information.

Cressman, K. 2013. Role of remote sensing in desert locust early warning. - J. Appl. Remote Sens. 7: 075098.

Cullen, D. A. et al. 2017. From molecules to management: mechanisms and consequences of locust phase polyphenism. - Adv. Insect Physiol. 53: 167-285.

Davidson, J. and Andrewartha, H. G. 1948. The influence of rainfall, evaporation and atmospheric temperature on fluctuations in the size of a natural population of Thrips imaginis (Thysanoptera). - J. Anim. Ecol. 17: 200-222.

Decesare, N. J. et al. 2012. Transcending scale dependence in identifying habitat with resource selection functions. - Ecology 22: 1068-1083.

Deveson, E. D. 2013. Satellite normalized difference vegetation index data used in managing Australian plague locusts. - J. Appl. Remote Sens. 7: 075096.

Deveson, E. D. and Walker, P. W. 2005. Not a one-way trip: historical distribution data for Australian plague locusts support frequent seasonal exchange migrations. - J. Orthoptera Res. 14: 91-105.

Deveson, E. D. et al. 2005. Evidence from traditional and new technologies for northward migrations of Australian plague locusts Chortoicetes terminifera (Walker) (Orthoptera: Acrididae) to western Queensland. - Austral Ecol. 30: 928-943.

Deveson, T. and Hunter, D. M. 2002. The operation of a GISbased decision support system for Australian locust management. - Entomol. Sin. 9: 1-12.

Dingle, H. 2014. Migration: the biology of life on the move. Oxford Univ. Press.

Ellis, P. E. and Ashall, C. 1957. Field studies on diurnal behaviour, movement and aggregation in the desert locust (Schistocerca gregaria Forskål). - Anti-locust bulletin. 25: 4-94.

FAO 2020. Locust hub. - <https://locust-hub-hqfao.hub.arcgis. $\mathrm{com} />$.

Farrow, R. A. 1979. Population dynamics of the australian plague locust, Chortoicetes terminifera (Walker), in Central-Western New-South-Wales I. Reproduction and migration in relation to weather. - Aust. J. Zool. 27: 717-745.

Farrow, R. A. 1990. Flight and migration in acridoids. - In: Biology of grasshoppers. Wiley, pp. 227-314.

Fauchald, P. and Erikstad, K. E. 2002. Scale-dependent predatorprey interactions: the aggregative response of seabirds to prey under variable prey abundance and patchiness. - Mar. Ecol. Prog. Ser. 231: 279-291.

Fauchald, P. and Tveraa, T. 2006. Hierarchical patch dynamics and animal movement pattern. - Oecologia 149: 383-395.

Fauchald, P. et al. 2000. Scale-dependent predator-prey interactions: the hierarchical spatial distribution of seabirds and prey. - Ecology 81: 773-783.

Forskål, 1775. Descriptiones Animalium Avium, Amphibiorum, Piscium, Insectorum, Vermium; quae in Itinere Orientall observati Petrus Forskal. Prof. Haun. Post morten Acutoris editt Carsten Nieburhr. 81.

Gorelick, N. et al. 2017. Google earth engine: planetary-scale geospatial analysis for everyone. - Remote Sens. Environ. 202: 18-27.

Greig-Smith, P. 1979. Pattern in vegetation. - J. Ecol. 67: 755-779.

Grinnell, J. 1917. The niche-relationships of the California thrasher. - Auk 34: 427-433.

Hanski, I. 1998. Metapopulation dynamics. - Nature 396: 41-49.

Hassler, S. K. et al. 2010. Vegetation pattern divergence between dry and wet season in a semiarid savanna - spatio-temporal dynamics of plant diversity in northwest Namibia. - J. Arid Environ. 74: 1516-1524.

Hunter, D. M. et al. 2001. Adaptations of locusts and grasshoppers to the low and variable rainfall of Australia. - J. Orthoptera Res. 10: 347-351.

Hunter, D. M. et al. 2008. Aerial detection of nymphal bands of the Australian plague locust (Chortoicetes terminifera (Walker)) (Orthoptera: Acrididae). - Crop Protect. 27: 118-123.

Ives, A. R. et al. 2008. High-amplitude fluctuations and alternative dynamical states of midges in Lake Myvatn. - Nature 452: 84-87.

Johnson, C. J. et al. 2004. A quantitative approach to conservation planning: using resource selection functions to map the distribution of mountain caribou at multiple spatial scales. - J. Appl. Ecol. 41: 238-251.

Johnson, D. H. 1980. The comparison of usage and availability measurements for evaluating resource preference. - Ecology 61: 65-71.

Jordahl, K. 2014. GeoPandas: python tools for geographic data. $<$ https://github.com/geopandas/geopandas $>$.

Kayalto, M. et al. 2020. Cartographie des zones de reproduction et de grégarisation du criquet pèlerin au Tchad. - Cahiers Agricult. 29: 14 .

Kearney, M. and Porter, W. 2009. Mechanistic niche modelling: combining physiological and spatial data to predict species' ranges. - Ecol. Lett. 12: 334-350.

Kearney, M. R. et al. 2014. Microclimate modelling at macro scales: a test of a general microclimate model integrated with gridded continental-scale soil and weather data. - Methods Ecol. Evol. 5: 273-286.

Kotliar, N. B. and Wiens, J. A. 1990. Multiple scales of patchiness and patch structure: a hierarchical framework for the study of heterogeneity. - Oikos 59: 253-260.

Levin, S. A. 1992. The problem of pattern and scale in ecology.Ecology 73: 1943-1967.

Macarthur, R. H. 1958. Population ecology of some warblers of northeastern coniferous forests. - Popul. Ecol. 39: 599-619.

Maestre, F. T. et al. 2021. Biogeography of global drylands. - New Phytol. 231: 540-558.

Mangeon, S. et al. 2020. Daily mapping of Australian Plague Locust abundance. - Sci. Rep. 10: 16915. 
Mao, D. et al. 2014. Spatiotemporal dynamics of grassland aboveground net primary productivity and its association with climatic pattern and changes in northern China. - Ecol. Indic. 41: 40-48.

Marra, G. and Wood, S. N. 2011. Practical variable selection for generalized additive models. - Comput. Stat. Data Anal. 55: 2372-2387.

McGarigal, K. et al. 2016. Multi-scale habitat selection modeling: a review and outlook. - Landscape Ecol. 31: 1161-1175.

Morlini, I. 2006. On multicollinearity and concurvity in some nonlinear multivariate models. - Stat. Methods Appl. 15: 3-26.

Morton, S. R. et al. 2011. A fresh framework for the ecology of arid Australia. - J. Arid Environ. 75: 313-329.

Murphy, E. J. et al. 1988. Scales of interaction between Antarctic krill and the environment. - In: Sahrhage, D. (ed.), Antarctic Ocean and resources variability. Springer, pp. 120-130.

Newman, E. A. et al. 2019. Scaling and complexity in landscape ecology. - Front. Ecol. Evol. 7: 293.

Noy-Meir, I. 1973. Desert ecosystems: environment and producers. - Annu. Rev. Ecol. Syst. 4: 25-51.

Noy-Meir, I. 1974. Desert ecosystems: higher trophic levels. Annu. Rev. Ecol. Syst. 5: 195-214.

Olson, D. M. et al. 2001. Terrestrial ecoregions of the world: a new map of life on Earth. - BioScience 51: 933-938.

Padilla, D. K. et al. 2014. Addressing grand challenges in organismal biology: the need for synthesis. - BioScience 64: 1178-1187.

Parry, H. R. 2013. Cereal aphid movement: general principles and simulation modelling. - Movem. Ecol. 1: 14.

Pedersen, E. J. et al. 2019. Hierarchical generalized additive models in ecology: an introduction with mgcv. - PeerJ 7: e6876.

Pedgley, D. 1980. Desert locust forecasting manual. - Centre for Overseas Pest Research.

Pedgley, D. E. 1973. Testing feasibility of detecting locust breeding sites by satellite. Final report to NASA on ERTS-1 experiment. - Centre for Overseas Pest Research.

Pettorelli, N. 2013. The normalized difference vegetation index. Oxford Univ. Press.

Pierson, E. A. and Turner, R. M. 1998. An 85-year study of saguaro Carnegiea gigantea demography. - Ecology 79: 2676-2693.

Piou, C. et al. 2013. Coupling historical prospection data and a remotely-sensed vegetation index for the preventative control of desert locusts. - Basic Appl. Ecol. 14: 593-604.

Piou, C. et al. 2017. Mapping the spatiotemporal distributions of the desert Locust in Mauritania and Morocco to improve preventive management. - Basic Appl. Ecol. 25: 37-47.

Rainey, R. C. 1963. Meteorology and the migration of desert locusts: applications of synoptic meteorology in locust control. Meteorology and the migration of desert locusts. Applications of synoptic meteorology in locust control. - Secretariat of the World Meteorological Organization.

Schwinning, S. and Sala, O. E. 2004. Climate change affects the outcome of competitive interactions - an application of principal response curves. - Oecologia 141: 211-220.

Senft, R. L. et al. 1987. Large herbivore foraging and ecological hierarchies: landscape ecology can enhance traditional foraging theory. - BioScience 37: 789-799.

Simpson, G. L. 2019. gratia: graceful 'ggplot'-based graphics and other functions for GAMs fitted using 'mgcv.' R package ver. 0.2-1. $-<$ https://cran.r-project.org/package=gratia $>$.
Stacey, G. 2017. Grand challenges for biological and environmental research: progress and future vision. - USDOE Office of Science (SC), Washington, DC (United States). Biological and Environmental Research (BER).

Sutherland, W. J. et al. 2013. Identification of 100 fundamental ecological questions. - J. Ecol. 101: 58-67.

Symmons, P. M. and Cressman, K. 2001. Desert locust guidelines: biology and behaviour. - The Food and Agriculture Organization.

Team, Q. D. 2020. QGIS geographic information system. - <www. qgis.org/en/site/>.

Thackway, R. and Cresswell, I. D. (eds) 1995. An interim biogeographic regionalisation for Australia: a framework for establishing the national system of reserves, Ver. 4.0, 4th edn. - Australian Nature Conservation Agency, Canberra.

Therville, C. et al. 2021. Locusts and people: integrating the social sciences in sustainable locust management. - Agronomy 11: 1-16.

Uvarov, B. 1977. Grasshoppers and locusts. A handbook of general acridology, vol. 2. Behaviour, ecology, biogeography, population dynamics. - Centre for Overseas Pest Research.

Uvarov, B. P. 1928. Locusts and grasshoppers: a handbook for their study and control. - Imperial Bureau of Entomology.

van Huis, A. 2007. Locust control by early identification of breeding sites. - Encyclopedia of pest management. Taylor and Francis, pp. 1-3.

Veran, S. et al. 2015. Modeling spatiotemporal dynamics of outbreaking species: influence of environment and migration in a locust. - Ecology 96: 737-748.

Walker, F. 1870. Catalogue of the specimens of Dermaptera saltatoria in the collection of the British museum 4: 777.

Wang, B. et al. 2019. Future climate change likely to reduce the Australian plague locust Chortoicetes terminifera seasonal outbreaks. - Sci. Total Environ. 668: 947-957.

Wardhaugh, K. G. 1986. Diapause strategies in the Australian plague locust (Chortoicetes terminifera Walker). - The evolution of insect life cycles. Springer, pp. 89-104.

Watt, S. A. 1947. Pattern and process in the plant community. - J. Ecol. 35: 1-22.

Van der Werf, W. et al. 2005. Plant communities can predict the distribution of solitarious desert locust Schistocerca gregaria. - J. Appl. Ecol. 42: 989-997.

White, F. 1983. The vegetation of Africa, a descriptive memoire to accompany UNESCO/AETFAT vegetation map of Africa; UNESCO: Paris, France.

Whitford, W. 2002. Conceptual framework and paradigms. - In: Whitford, W. and Duval, B. (eds), Ecology of desert systems. Academic Press, NY, USA.

Wickham, H. 2018. Tidyverse: easily install and load the 'Tidyverse'. 2017. R package ver. 1.2.1. - <https://cran.r-project.org/ package $=$ tidyverse $>$.

Wiens, J. A. 1989. Spatial scaling in ecology. - Funct. Ecol. 3: 385-397.

Wood, S. N. 2017. Generalized additive models: an introduction with R. - Chapman and Hall/CRC.

Wood, S. N. et al. 2016. Smoothing parameter and model selection for general smooth models. - J. Am. Stat. Assoc. 111: $1548-1563$.

Wu, J. and Loucks, O. L. 1995. From balance of nature to hierarchical patch dynamics: a paradigm shift in ecology. - Quart. Rev. Biol. 70: 439-466. 